\title{
Les TIC dans les collectifs diasporiques : étude des Bretons à New York
}

\section{Simon LE BAYON}

\section{Q OpenEdition \\ 1 Journals}

Édition électronique

URL : http://journals.openedition.org/ticetsociete/695

DOI : 10.4000/ticetsociete.695

Éditeur

Association ARTIC

\section{Référence électronique}

Simon LE BAYON, « Les TIC dans les collectifs diasporiques : étude des Bretons à New York »,

tic\&société [En ligne], Vol. 3, n 1-2 | 2009, mis en ligne le 20 avril 2019, consulté le 20 avril 2019. URL http://journals.openedition.org/ticetsociete/695; DOI : 10.4000/ticetsociete.695 
tic\&société - 3 (1-2), 2009

\title{
Les TIC dans les collectifs diasporiques : étude des Bretons à New York
}

\author{
Simon Le Bayon
}

LAS EA2241

Université européenne de Bretagne, Rennes II

Zac Atalante Champeaux

3 allée Adolphe Bobierre, 35000 Rennes

slebayon@gmail.com

Simon Le Bayon est doctorant au Laboratoire d'Anthropologie et de sociologie de l'Université de Rennes II. Dans le cadre d'une convention d'insertion et de formation par la recherche en entreprise (Cifre), il intervient en tant qu'animateur d'un réseau diasporique pour la société Zindep. 


\title{
Les TIC dans les collectifs diasporiques : étude des Bretons à New York
}

\begin{abstract}
Résumé
Dans cet article, nous nous intéressons au collectif de la diaspora bretonne à New York. En tant que réseau de connaissances diasporiques, ce collectif d'expatriés agit pour le développement de son pays d'origine. Nous étudierons ici les usages des technologies de l'information et de la communication (TIC) opérés par ce collectif, au travers des traces numériques. Cette analyse nous permet de caractériser trois phases d'activités successives qui correspondent à différents régimes d'engagement. Notre analyse met en lumière les interactions qui existent entre les outils techniques, le type de relation à l'intérieur du collectif et les connaissances produites par cet ensemble.
\end{abstract}

Mots-clés : Diaspora, internet, médiation, collectif, Breton.

\begin{abstract}
In this paper, we focus on a group of Breton people settled in New York City. This diaspora knowledge network is acting for the development of its country of origin. We focus on this collectivity's uses of information and communication technologies by examining their logs. This analysis describes three different stages that correspond to different régimes d'engagement. Our analysis highlights intereactions between technological tools, the types of relationships within the collectivity and the knowledge produced by the group.
\end{abstract}

Keywords : Diaspora, Internet, mediation, community, Breton.

\section{Resumen}

Este artículo se interesa en el colectivo de la diáspora bretona en Nueva York. En tanto que red de conocimiento diaspórico, este colectivo de expatriados actúa en favor del desarrollo de su país de origen. A través de rastros digitales, se estudian los usos de las tecnologías de la información y de la comunicación (TIC) utilizadas por dicho colectivo. Este análisis nos permite caracterizar tres fases de actividades sucesivas que corresponden a los diferentes regímenes de compromiso. Tal análisis arroja luz sobre las 
Les TIC dans les collectifs diasporiques : étude des Bretons à New York

interacciones existentes entre las herramientas técnicas, el tipo de relación existente en el interior del colectivo y los conocimientos producidos por este conjunto.

Palabras clave : Diáspora, internet, mediación, colectivo, Bretone. 


\section{Introduction : diasporas et usages de l'internet}

Jean-Baptiste Meyer (Meyer, 2007) note, depuis le début des années 2000, une explosion du nombre de sites web animés par des migrants et visant à promouvoir le développement de leur pays d'origine. Ces réseaux de connaissances diasporiques, ou diaspora knowledge networks, sont formés par des expatriés avec de hauts niveaux de compétence. Ces expatriés contribuent au développement de leur territoire d'origine par la mise en commun de leurs réseaux respectifs. Bien que ces réseaux de connaissances diasporiques soient particulièrement présents dans les programmes de coopération entre pays du Nord et du Sud, il en existe aussi entre pays développés. Dans cet article nous évoquerons la diaspora bretonne ${ }^{1}$. Les membres des réseaux de connaissances diasporiques et plus généralement des diasporas développent une forte connectivité technique pour « maintenir à distance et activer quotidiennement des relations » (Diminescu, 2005). Nos travaux sur des collectifs de la diaspora bretonne (Boullier, Le Bayon et Philip, à paraître) constituent une opportunité pour questionner l'activité et la cognition dans ces formes particulières de " collectifs éclatés » (Borzeix et Cochoy, 2008).

Depuis mars 2006, dans le cadre d'une activité professionnelle, j'anime un réseau visant à mettre en relation des entrepreneurs bretons avec leurs compatriotes de l'étranger. Cette activité me donne une place privilégiée pour observer comment de nouveaux collectifs de Bretons se constituent dans différents pays du monde et participent à différents projets ${ }^{2}$. Les collectifs de la diaspora bretonne se développent sous des formes variées tant en termes d'effectifs, d'activités et d'organisations. Néanmoins ils partagent tous des usages avancés et pluriels des technologies de l'information et de la communication (TIC). Dans cet article, nous nous intéressons à la diaspora bretonne de la ville de New York.

Entre mars 2006 et novembre 2008, nous avons suivi les activités de ce collectif principalement au travers des traces laissées dans les supports numériques que sont les sites web et les échanges de mèls. Bien entendu, l'activité de ce collectif ne se réduit pas à ces traces. Cependant, pour l'objet de cet article, nous focaliserons notre attention sur les seules médiations électroniques, c'est-à-dire les dispositifs techniques qui «transforment,

\footnotetext{
1 La Bretagne est une région administrative située à l'extrémité ouest de la France, elle est composée de quatre départements (Côtes d'Armor, Finistère, Ille et Vilaine, et Morbihan).

${ }^{2}$ Dans la suite du texte, les lettres SLB font référence à ma position d'animateur de réseau.
} 
Les TIC dans les collectifs diasporiques : étude des Bretons à New York

traduisent, distordent et modifient le sens ou les éléments qu'ils sont censés transporter » (Latour, 2006, p.58).

Les traces récoltées au cours des activités nous permettent de caractériser trois phases. Ces phases constituent des moments pendant lesquels le collectif déploie son énergie dans un mode d'action en commun. Le collectif, qui, comme nous le verrons, est en mouvement permanent, ne peut mener en même temps ces trois modes d'action. Pour chacune de ces phases, la forme du collectif, les outils présents et les connaissances produites prennent une configuration particulière. Nous allons montrer comment, dans une première phase, l'activité est guidée par la découverte et la mise en relation d'un collectif très large, puis comment, dans une seconde phase, le collectif d'expatriés exploite l'organisation d'événements pour gagner en visibilité et en autorité dans son pays d'origine, et enfin comment, dans une troisième phase, les activités sont caractérisées par la consolidation des liens entre les membres.

\section{Comment construire un réseau diasporique ?}

\subsection{La méthode d'exploration d'un jeune Breton à New York}

Obala, trentenaire, s'installe dans la région de New York, avec sa femme et leur fils, en décembre 2005. Au cours des dix dernières années, Obala a travaillé dans le génie civil en Côte d'Ivoire puis à Shanghai. II a ensuite commencé une carrière de commercial au Mexique. À chaque arrivée dans un nouveau pays, Obala s'est appuyé sur la diaspora bretonne pour trouver une activité. Arrivant sans emploi aux États-Unis, il cherche des Bretons afin de détecter et de saisir des opportunités d'activités. Dans une interview de décembre 2006, Obala explique :

«Dès le départ, je me suis affirmé et présenté en tant que Breton. Quand tu es jeune [...] tu trouveras toujours sur ta route un Breton plus âgé prêt à te filer un coup de main. Tu lui ramènes l'image de sa jeunesse et de sa Bretagne. L'identité bretonne facilite le rapport, l'échange est beaucoup plus direct et facile entre Bretons. [...] En se rencontrant, on ouvre les portes de nouveaux réseaux, on multiplie les contacts et les opportunités ${ }^{3}$. »

\footnotetext{
3 Interview disponible à l'adresse suivante : http://institut-locarn.com/diaspora/blog.2006-0303.8745537556/blogentry.2007-12-05.7855752998, consulté le 18/12/2008.
} 
Mais, à New York, la présence des Bretons reste discrète. Excepté le club sportif du "Stade Breton », il n'y a que dans les livres et les mémoires qu'Obala identifie des traces de la Bretagne. Dans les années 1960, l'« Association Bretonne ", tombée en désuétude depuis, réunissait plusieurs centaines de personnes au cours de bals, pique-niques et manifestations sportives (Jouas, Le Corre et Jamet, 2005). Obala se donne pour objectif de raviver l'esprit des Bretons de New York. Pendant plusieurs mois, au gré des personnes qu'il rencontre et des outils qu'il utilise, Obala tisse un large réseau social de Bretons. Bien qu'il vive à proximité de New York, Obala n'y limite pas ses recherches. Les outils techniques viennent accroître ses capacités à identifier de nouvelles personnes et à échanger avec elles.

\subsection{Les technologies d'exploration}

\subsubsection{Les forums francophones de New York}

À travers Skype ${ }^{4}$, Obala retrouve Éric, un ami rencontré à Mexico et qui vit maintenant à New York. Pour Éric, la quête d'Obala représente une opportunité, à la fois personnelle et professionnelle. En effet, Éric est un Breton de Nantes qui travaille pour une société de gestion de patrimoine et les Bretons, ainsi que tous les Français expatriés aux États-Unis, font partie de sa cible commerciale. Éric va donc s'investir pour construire avec Obala un réseau de Bretons à New York. On retrouve des traces numériques laissées par Éric sur le site web "Entre New York ", qui se présente comme le "forum de la communauté francophone à New York ». Le 23 mai 2006, dans la catégorie "Rencontre du Forum ", Éric crée le sujet "Association des Bretons de New York Rencontre. » et y publie le message suivant :

"Chers amis [...] anciens et nouveaux Bretons de New York sont conviés à nous rejoindre lors du prochain événement qui aura lieu le Dimanche 4/06 à $17 \mathrm{H} 00$ au TOUT VA BIEN. Nous aurons le plaisir de retrouver certains anciens faisant partie du STADE BRETON, et nous serons accueillis par Jean-Pierre et son fils Mickael. [...] Afin de pouvoir prévoir le nombre

\footnotetext{
${ }^{4}$ Skype est un logiciel de voix sur internet (Voice Over Internet Protocol) qui permet d'utiliser son ordinateur comme un téléphone. La communication vers d'autres ordinateurs équipés de Skype est gratuite.

5 Entre New York, site web disponible à l'adresse http://entrenewyork.com/, consulté le 20 septembre 2008
} 
Les TIC dans les collectifs diasporiques : étude des Bretons à New York

de participants, nous vous remercions de bien vouloir confirmer votre venue avant le 2/06. Kenavo »

À la suite de cette annonce, seize personnes vont échanger une cinquantaine de messages sur une période de deux mois et demi. Les discussions sur le forum connaissent des pics d'activités à proximité des deux repas organisés durant le mois de juin, puis du pique-nique prévu à Central Park fin juillet. Sur ce fil de discussion, Éric est l'auteur de plus d'un tiers des messages, il répond personnellement aux commentaires et il accueille les nouveaux membres. Au travers des échanges, on distingue deux styles de communication. Les habitués du forum ont tendance à faire des digressions à partir du thème de la Bretagne. Ils discutent du nom des départements bretons, de l'histoire du drapeau breton ou encore de l'appartenance du département de la Loire-Atlantique à la Bretagne en tant que région administrative. Les habitués du forum se répondent les uns les autres, parfois dans un laps de temps très court qui fait oublier qu'ils exploitent un outil d'échange asynchrone. Les nouveaux inscrits du forum se caractérisent par un style d'échange plus utilitaire. Ils répondent aux invitations pour les repas ou demandent des précisions, sans rebondir sur les digressions. Éric jongle entre les styles, apportant des réponses adaptées et personnalisées.

Au fil des annonces, l'équipement technique d'Éric s'enrichit. Alors que le premier message invite les personnes intéressées à confirmer leur participation directement sur le forum, un second message invite à répondre sur l'adresse mèl personnelle d'Éric. Par la suite, une adresse collective, bzhreseau@yahoo.com, est donnée en référence pour les inscriptions. Celles et ceux qui écrivent à cette adresse sont ajoutés à une liste de diffusion pour être informés des activités de ce nouveau collectif.

On assiste à une spécialisation progressive des outils. Initialement, l'outil forum devait couvrir tous les besoins liés à l'organisation d'un événement. L'introduction du mèl permet de filtrer les inscriptions aux événements organisés. Les discussions et digressions devant rester quant à elles sur le forum. En contrepartie, le fil de discussion s'appauvrit. Ensuite, l'adresse collective est créée pour mettre en commun les démarches d'Obala et d'Éric. Enfin, la liste de diffusion répond à une autre problématique : toucher plus largement les membres du réseau et pas seulement ceux présents sur ce forum. Par la suite, les messages déposés sur les forums annonçant les prochains événements du collectif susciteront moins de réactions et d'échanges. 


\subsubsection{Les services de réseaux sociaux}

En mars 2006, Obala s'inscrit sur le service de réseau social en ligne Viadéo, " plateforme de mise en relation professionnelle [...] qui permet d'enrichir et de capitaliser sur son réseau des relations professionnelles ; et par conséquent d'augmenter ses opportunités de business et de développement de carrière ${ }^{6}$ ". Avec Viadéo, Obala découvre le forum «Bretagne > bzhnetwork». Sur ce forum, des "Bretons et amis de la Bretagne ", à la fois en Bretagne et à l'étranger, partagent des informations et des actualités sur le thème de la Bretagne. C'est au travers de ce forum qu'Obala rencontre SP, créateur et animateur du forum, qui vit au Japon. Par l'intermédiaire de SP, Obala prend contact avec GB, SLB et EC. Ces trois personnes sont membres de l'Institut de Locarn $^{7}$, une association qui réunit des chefs d'entreprises en Bretagne. Cet institut a mis en place le réseau "Diaspora Économique Bretonne " dont l'objectif est de mettre en relation des entrepreneurs de Bretagne avec des Bretons expatriés afin de participer ensemble au développement économique des entreprises de Bretagne. Obala détecte rapidement la complémentarité des réseaux Diaspora Économique Bretonne et BZH Network. Par sa position, Obala identifie aussi l'attrait qu'il peut représenter pour les porteurs de ces projets.

La figure 1 illustre la mise en relation de ces différents acteurs. Elle est réalisée à l'aide du logiciel de cartographie de réseaux hétérogènes RéseauLu. Nous avons utilisé ce logiciel pour explorer un corpus de 450 messages mèl et pour les représenter sous forme de graphes. Ce corpus a été collecté au cours du suivi en temps réel des activités du collectif des Bretons de New York entre mars 2006 et novembre 2008. La construction et l'exploration des cartes nous ont aidé à identifier les phases que nous décrivons. La figure 1 est représentative de la phase d'extension de réseau. Tous les points représentent des adresses mèls et les arcs sont calculés à partir du nombre d'échanges de messages. Plus les points sont proches plus le nombre de messages échangés est important. Plus le point est gros plus cette adresse mèl est à l'origine d'un nombre important de messages. Ici nous n'avons positionné sur la carte que les noms des acteurs cités dans l'article. Sur cette première phase qui va de mars à

\footnotetext{
${ }^{6}$ Disponible à : http://www.viadeointhenews.com/francais/press/view.asp?id=2\&pressid=63, consulté le 18 décembre 2008.

Institut de Locarn, cultures et stratégies internationales. Site web disponible à l'adresse: http://institut-locarn.com, consulté le 18 décembre 2008

${ }^{8}$ RéseauLu est un logiciel et un système analytique qui réunit dans un environnement analytique différents types de données quantitatives et qualitatives. Conception, développement Andrei Mogoutov, www.aguidel.com.
} 
octobre 2006, les principaux auteurs de messages, représentés par des ronds, sont EC et SP. Les carrés sont les destinataires. Ce schéma nous montre comment Obala passe par le réseau BZH Network de Viadéo pour ensuite découvrir le réseau de l'Institut de Locarn, représenté ici par le cercle formé autour d'EC.

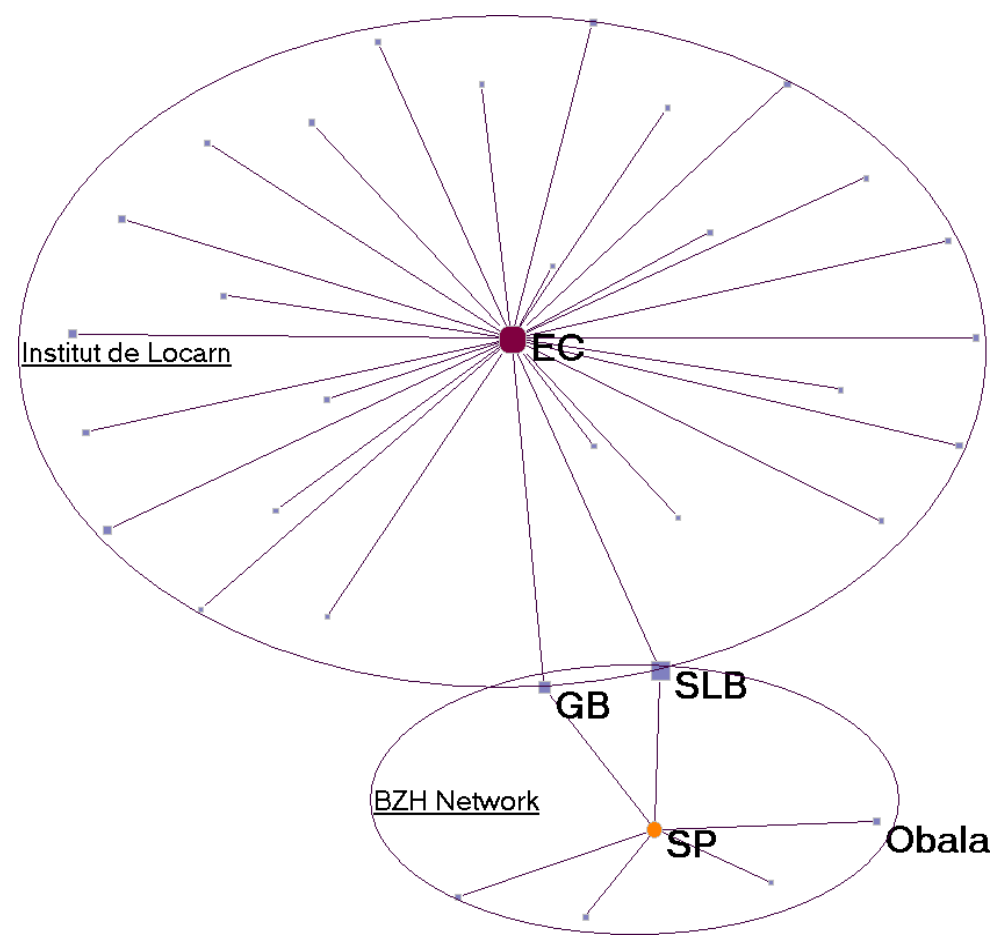

Figure 1 : Obala à New York découvre et prend contact avec l'Institut de Locarn en Bretagne, par le biais du réseau BZH Network de Viadéo

Alors qu'Obala se lançait dans la constitution d'un nouveau réseau de Bretons aux États-Unis, Skype et Viadéo le font aller à la rencontre d'un public beaucoup plus vaste. Obala procède par tâtonnement, sans suivre un plan établi à l'avance. Son activité, pragmatique, est guidée par la rencontre et la recherche de nouveautés. Ces éléments sont caractéristiques de ce que Nicolas Auray nomme le régime d'exploration (Auray, 2005). Ce régime d'exploration est guidé par l'excitation liée à la découverte d'une nouveauté. II s'intègre dans le modèle plus large de ce que Laurent Thévenot caractérise comme les régimes d'engagement (Thévenot, 2006). 
Obala est dans une démarche pragmatique, il s'adapte en permanence aux circonvolutions du parcours pour nouer de nouvelles associations et rebondir sur de nouvelles opportunités. Sur Viadéo, la rencontre avec SP puis avec les membres de l'Institut de Locarn sont des opportunités qu'Obala saisit. Un réseau actif de Bretons à New York doit pouvoir intéresser des entreprises bretonnes. Toujours sur Viadéo, Obala met en avant sa compétence exploratoire dans ses quelques contributions sur le forum. II y démontre sa capacité à identifier et à connaître des Bretons tout autour de la planète.

\subsubsection{Un service de VolP, Skype}

Pour retrouver les traces des Bretons à New York, Obala identifie les noms de familles populaires en Bretagne sur les sites web de généalogie. Puis, avec ces noms, il lance des requêtes combinées avec des indices territoriaux (New York, USA, New Jersey...) sur les moteurs de recherche du web. Avec ces mêmes noms, il lance aussi des requêtes sur le moteur de recherche de Skype. Pour Obala, qui se présente lui-même comme très peu à l'aise avec l'écrit, Skype permet de prendre contact oralement et surtout de transmettre son énergie et son dynamisme de façon beaucoup plus efficace qu'à l'écrit. Obala est guidé par une logique d'exploration dans laquelle la découverte de nouveautés permet de maintenir un état permanent d'excitation (Auray, 2005). Skype s'avère être une formidable ressource pour cette exploration. La façon dont Obala exploite cet outil lui permet d'accroître significativement le nombre de relations mais aussi la découverte de nouvelles personnes et de nouveaux projets. Pour Obala, Skype est encore plus puissant que ne le sont les services de réseaux sociaux. Les relations synchrones de Skype en communication orale et écrite alimentent une immédiateté favorable à l'excitation. Par exemple, les icônes qui représentent chaque contact du carnet d'adresse indiquent un état : en ligne, disponible, occupé... Ce repère permet d'entrer en communication avec un contact dès qu'il allume son ordinateur. Le carnet d'adresse Skype de plus de trois cents contacts donne une idée de l'importance de cet outil dans la démarche d'Obala.

Toutes les technologies de l'internet ne sont pas des technologies de l'exploration. Un outil ne devient une ressource d'exploration qu'une fois combiné avec un usage. Dans nos exemples, le forum utilisé par Éric devient difficilement une technologie de l'exploration. Skype, particulièrement bien équipé avec ses fonctions d'échanges synchrones, devient, par l'usage qu'en fait Obala, une ressource efficace de l'exploration. 
Les TIC dans les collectifs diasporiques : étude des Bretons à New York

\subsection{Un collectif étendu, construit par des outils techniques}

Pour tisser ce nouveau réseau, les acteurs ont mobilisé leurs outils favoris. Ces outils, en bons médiateurs (Latour, 2006), participent activement à la forme du réseau. Dans un cas, Obala utilise massivement Skype qui lui permet d'être présent en quasi-permanence avec ses contacts. Les procédures et les modes opératoires qu'il a développés en font une ressource de l'exploration. Pour Obala, Skype offre un « vivier luxuriant et foisonnant de contacts potentiels et de nouvelles formes d'associations » (Auray, 2005, p.4).

Dans un second cas, la rencontre avec SP sur Viadéo permet à Obala d'identifier et d'intégrer de nouveaux réseaux, ici un réseau d'entrepreneurs en Bretagne avec l'institut de Locarn. Mais Viadéo ne permet pas aussi bien que Skype de maintenir cet état de présence.

Dans un troisième cas, Éric utilise le forum francophone de New York pour annoncer des événements. Mais sur le forum, la nouveauté n'est pas particulièrement présente, ce sont les habitués qui s'expriment. L'analyse des discussions inscrit le forum dans un régime de "justification en public » (Thévenot, 2006). Le régime de justification en public vise à s'assurer que les participants partagent un même ensemble de principes généraux. Ces principes, ou conventions, vont asseoir la coordination et les liens entre les personnes. Le régime de justification permet aux membres d'un groupe de s'assurer qu'ils agissent au nom d'une même cause. Les messages déposés par Éric mettent en avant la convivialité du lieu, le repas partagé, et les liens quasi-familiaux avec les anciens Bretons de New York. Sur le forum, les interactions sont localisées et personnalisées.

L'analyse lexicale des échanges fait ressortir les champs sémantiques de la convivialité, de la politesse. Ces champs sémantiques répondent aux figures présentées par Obala dans son interview : le «Breton plus âgé », la répétition du voyage par les jeunes générations et la notion de confiance créée par l'attachement à la Bretagne. Tous ces éléments permettent de préciser le régime de justification qui prévaut sur ce fil de discussion. La valeur mise en avant, celle qui fait convention, est une valeur domestique ou familiale (Boltanski et Thévenot, 1991). Dans leur ouvrage De la justification, Luc Boltanski et Laurent Thévenot présentent six conventions au nom desquelles la coordination peut se réaliser lors d'un conflit ou d'une controverse. La convention domestique érige en principe le respect de la tradition, la confiance et les liens familiaux. On retrouve de façon nette ce modèle de valeur sur le 
forum : le repas, le caractère familial de l'établissement, et le Stade Breton, symbole de la génération précédente des Bretons de New York.

L'exploration entreprise par Obala et Éric construit un collectif particulièrement étendu. Le réseau des Bretons aux États-Unis regroupe des personnes présentes physiquement sur les cinq continents et pas uniquement à New York. Le collectif ainsi constitué est totalement déconnecté des instances officielles bretonnes. L'attachement entre les membres de ce collectif repose plus sur une identité partagée que sur un lien formel de nationalité ou de présence sur un territoire. Ces éléments sont caractérisés par Stéphane Dufoix, dans son ouvrage Les Diasporas, par ce qu'il appelle un mode " atopique de structuration de l'expérience collective à l'étranger » (Dufoix, 2003).

Dans cette première partie, nous avons suivi un regroupement d'acteurs qui ont en commun un attachement à la Bretagne. Les outils internet mobilisés agissent dans la constitution du collectif, en ouvrant grand les possibilités de rencontre et de découverte. La dynamique du collectif est guidée par l'exploration et la connectivité des membres ; il s'agit d'engranger toujours plus de contacts, de créer du lien pour, peut-être à l'avenir, réaliser un projet.

\section{Organiser un événement pour gagner en visibilité}

\subsection{La présence bretonne dans un événement majeur à New York}

La grande parade de la Saint-Patrick, fête nationale irlandaise, réunit tous les ans à New York plusieurs centaines de milliers de participants. C'est un événement majeur pour la population new yorkaise auquel les personnalités politiques et médiatiques s'associent. Pour la $246^{\mathrm{e}}$ édition, en mars 2007, le groupe "Kevrenn Alré », un ensemble de musique et de danse d'inspiration traditionnelle bretonne, est invité à participer au défilé. Bob, membre du bureau de Kevrenn Alré, en charge des relations internationales, souhaite optimiser le déplacement d'une cinquantaine de musiciens pour promouvoir sur place la culture et la création bretonne. Pour couvrir les frais, estimés à $75000 €$, Kevrenn Alré espère associer des partenaires privés qui pourront jouir d'une publicité lors des concerts. Bob dispose de quelques mois pour assurer l'organisation des concerts et le financement du déplacement. C'est en recherchant des contacts aux États-Unis qu'il entre en relation avec Obala et le nouveau groupe de Bretons. À New York, Kevrenn Alré a besoin d'une équipe qui puisse l'aider à organiser deux concerts. 
Les TIC dans les collectifs diasporiques : étude des Bretons à New York

À partir de novembre 2006, un petit groupe émerge du réseau des Bretons des États-Unis. Ce groupe, composé d'une dizaine de personnes, parmi lesquelles Obala et Éric, s'engage à accueillir Kevrenn Alré et à organiser plusieurs concerts. Ce nouveau collectif se désigne sous le nom de "Bzh-NY » et d'un logotype. Pendant quatre mois, les membres de Bzh-NY vont mener de front les échanges avec le comité organisateur de la parade, trouver et réserver des salles, promouvoir les concerts. Mais ce groupe ne va pas se contenter d'être actif à New York. Même en Bretagne, Bzh-NY est présent aux côtés de Kevrenn Alré dans des articles de presse, des interventions lors de colloques et séminaires, et lors de rencontres avec les représentants d'associations professionnelles et d'organismes publics économiques.

\subsection{Des outils de communication vers le public}

\subsubsection{L'utilisation particulière du mèl}

Pendant quelques mois, Obala communique de façon intensive par mèls. II exploite sa messagerie électronique à la façon d'un émetteur relais. Obala fait suivre à certains membres de son carnet d'adresse des messages et des discussions dont il n'est pas l'auteur. II bascule ainsi les contenus et les documents attachés en pièces jointes d'un réseau vers un autre. Obala transmet par exemple des discussions et des documents rédigés par des membres de Bzh-NY vers le collectif BZH Network. Sur la période du 31 mai 2006 au 30 janvier 2007, 55\% des messages envoyés par d'Obala sur cette liste comportaient la mention (faute d'orthographe incluse), «Remarque : message transférer en pièce jointe ».

Cet indice réfute toute intention d'Obala de reprendre à son compte le contenu, il se positionne explicitement comme relais. Sa position, à la jonction de plusieurs réseaux, lui permet de contrôler les informations qui passent d'un réseau à l'autre. Sa multi-appartenance se retrouve affirmée dans la signature de ses mèls, où il indique son statut d'« Initiateur de www.Bzh-ny.org et de Cofondateur de www.bzhnetwork.com ». En diffusant largement et régulièrement de l'information, Obala se positionne en "point de passage obligé " (Callon, 1986) entre la Bretagne et New York. Au travers des mèls, et plus largement des articles de presse et des rencontres, Obala soulève la question des promoteurs des produits et services bretons aux États-Unis. La diaspora bretonne constitue aussi une porte d'entrée vers les réseaux entrepreneuriaux. Ce discours soulève d'ailleurs quelques réactions, en Bretagne, de la part des professionnels de l'accompagnement à l'étranger. 


\section{Simon LE BAYON}

Nous pouvons illustrer cette situation par la figure 2. Cette carte est construite à partir d'échanges de mèls reçus par l'animateur du réseau Diaspora Économique Bretonne d'octobre 2006 à mars 2007. Construite à partir de 175 mèls échangés à propos de Bzh-NY, cette carte ne prétend pas à l'exhaustivité des échanges. Ici encore les carrés représentent les destinataires de messages et les ronds les auteurs. Dans cette carte, Obala est maintenant au coeur d'un large réseau d'émission de messages. L'événement constitue une opportunité pour contacter directement des acteurs en Bretagne. En procédant ainsi, il attire vers lui certains acteurs qui appartenaient au réseau « Institut de Locarn ».

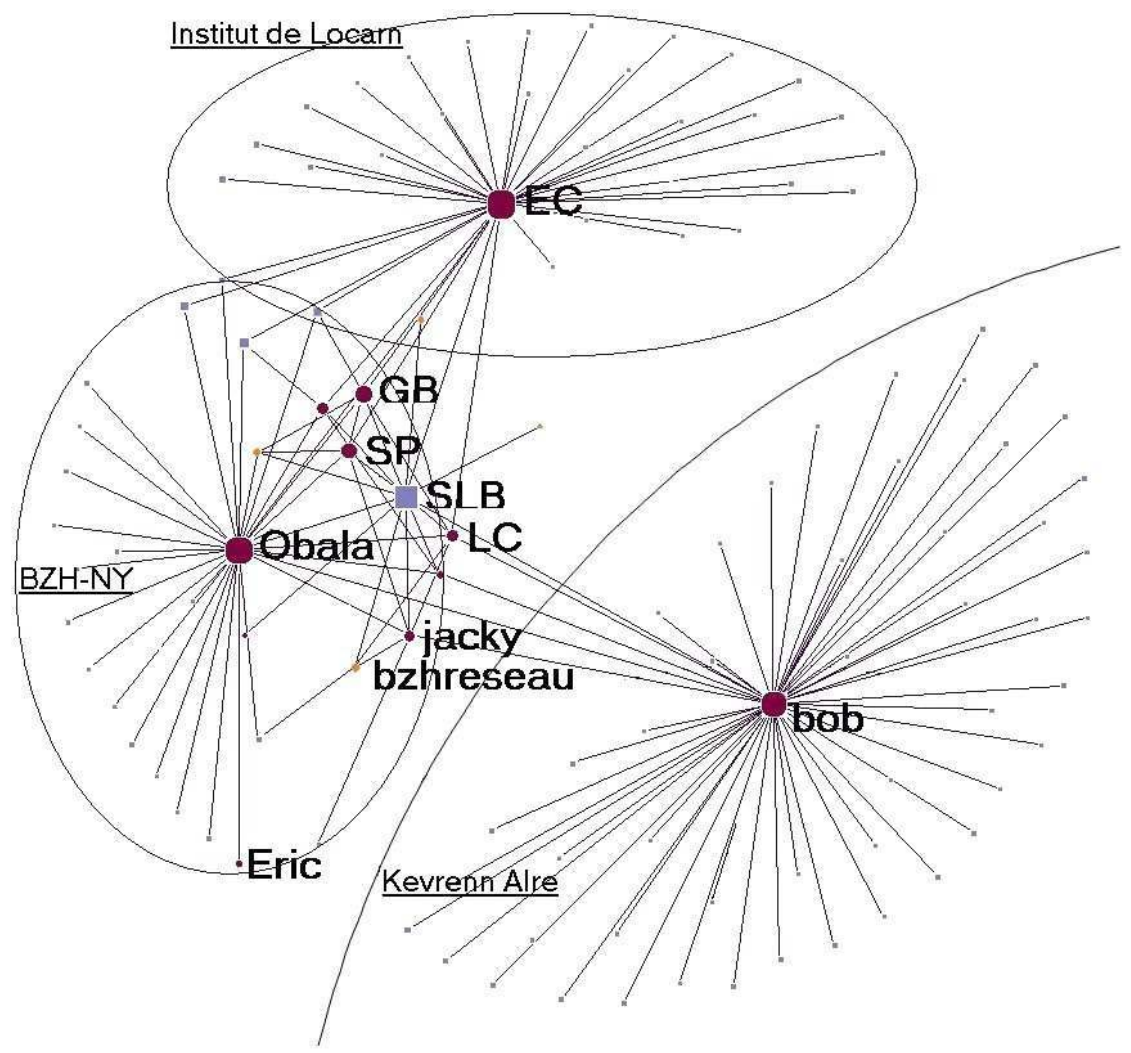

Figure 2 : Obala tire vers lui les acteurs du réseau Institut de Locarn 
Les TIC dans les collectifs diasporiques : étude des Bretons à New York

\subsubsection{La diffusion des documents bureautiques}

Cette même phase est marquée par la multiplication de documents bureautiques en circulation. Des fichiers informatiques viennent peupler les échanges des membres de Bzh-NY. On retrouve des tableurs Excel contenant les coordonnées d'associations françaises à New York, des contacts presse en France, ou encore des listes de tâches à effectuer. On retrouve aussi des diaporamas sur lesquelles sont schématisés des plannings. Ces documents servent de point de départ à des discussions lors des échanges par mèls et en cela ils constituent des ressources de coordination. Le collectif met en forme des connaissances, produit des outils de mesure et d'organisation pour accomplir au mieux le projet à venir. Ce mode d'activité est défini par Laurent Thévenot comme le régime de l'action en plan (Thévenot, 2006). Dans ce régime, l'individu est porteur d'un projet, et il va mettre en œuvre des ressources pour obtenir satisfaction en menant à bien son projet, en collaboration avec une communauté. La prolifération de documents de coordination accompagne la nouvelle activité du collectif. L'implication des membres dans cet événement s'accompagne d'une réduction du collectif que l'on a caractérisé dans la phase d'exploration. Les « liens faibles » (Granovetter, 1973) tissés par Obala et Éric lors de la phase d'exploration laissent place à des liens beaucoup plus forts qui se construisent dans l'action en commun. À la manière de ce qu'Etienne Wenger identifie dans les communautés de pratique (Wenger, McDermott et Snyder, 2002), le réseau lâche et étendu est un terrain fertile pour donner naissance à un « noyau dur ». Ce noyau, composé d'une dizaine de personnes, fixe les réunions, en donne l'orientation et y participe. C'est aussi ce groupe qui réalise les différentes tâches.

D'autres documents viennent agrémenter les échanges. Ce sont des diaporamas et des dossiers destinés aux potentiels sponsors en Bretagne. Ces dossiers de sponsoring présentent les enjeux de l'événement et l'opportunité pour une entreprise d'appuyer sa stratégie de développement à l'international sur des événements culturels. Les dossiers de sponsoring remis en mains propres ou diffusés par mèl constituent des clés pour intégrer de nouveaux réseaux. Les présentations Powerpoint sont projetées lors d'assemblées à des entrepreneurs et élus bretons. Ces mêmes présentations sont ensuite distribuées par voie électronique à l'assistance. Les documents bureautiques deviennent des tenant-lieux ou des « doublures » (Boltanski et Chiapello, 1999, p.448) pour les explorateurs de réseau que sont les membres de Bzh-NY lorsqu'ils recherchent des partenaires en Bretagne. Remis sous forme de tirage papier les documents font valoir leur immobilité. Les acteurs se déplacent pour 
présenter l'initiative à un maximum de personnes, et derrière eux, ils laissent ces documents immobiles.

\subsubsection{Un site web vitrine}

En décembre 2006, le site web Bzh-ny. org $^{9}$ est publié. C'est Jack, par ailleurs webmaster du site de "l'association de défense de la langue bretonne aux États-Unis », qui a proposé ses compétences. Jusqu'alors, ces acteurs n'ont utilisé que des outils publics et partiellement gratuits. Le site web est construit à l'aide d'un éditeur de page web et d'une base de données. Le site web n'exploite pas un CMS (système de gestion de contenu) en ligne. Le webmaster utilise un éditeur de code $\mathrm{html}$ pour construire le site sur son ordinateur personnel. Une fois modifié, le site est transféré vers la base de données du serveur web. Ce type de technologie restreint la dimension collaborative du site web car seul le webmaster est à même d'apporter des modifications par le biais des logiciels qu'il utilise.

Le site est construit pour la promotion de la semaine de festivités. II offre la possibilité de commander et de payer en ligne des CD de Kevrenn Alré. Le programme des festivités de la semaine est affiché. Pour quelques-uns des événements, il est possible de s'inscrire par formulaire et de régler la participation en ligne. Alors qu'auparavant, ces actions étaient réalisées manuellement par Obala et Éric, désormais le site web les réalise de façon quasi automatique. Le choix de l'architecture du site web n'est pas anodin (Boullier, 2008) : ce type de technologie asynchrone et caractéristique du « web 1.0 " n'est pas aligné avec la logique d'exploration de réseau que l'on a rencontrée dans la première phase. Ici, le site web est subordonné à l'opération de communication que constitue le déplacement de Kevrenn Alré. Le site web, vitrine de l'événement, a été guidé par la recherche de visibilité et le désir d'être reconnu, comme peuvent le montrer les pages "revue de presse» et "partenaires ». Ces éléments nous renvoient vers un régime de justification en public basé sur le principe de l'opinion (Boltanski et Thévenot, 1991). Dans ce mode de justification, le renom, la réputation, être au coeur des discussions du grand public sont les valeurs à partir desquelles les acteurs se coordonnent. L'agencement du site est conçu pour que les logos des sponsors de l'association soient toujours visibles. L'architecture du site web, en particulier l'absence d'outils de communication ou de discussion, renforce la démarcation entre le collectif organisateur et son public.

\footnotetext{
${ }^{9}$ Disponible à l'adresse http://Bzh-ny.org, consulté le 15 décembre 2008.
} 
Les TIC dans les collectifs diasporiques : étude des Bretons à New York

\subsubsection{Newsletters et listes de diffusion}

Le contrat d'hébergement du site web offre la possibilité d'utiliser des listes de diffusion. Cet outil est utilisé pour diffuser une newsletter à l'ensemble des adresses mèls collectées depuis plusieurs mois. Les newsletters contiennent des informations sur les activités du réseau, dont deux soirées de promotion en décembre 2006 et en janvier 2007. Sur le même principe technologique, des listes de diffusion "presse " regroupent les coordonnées de journalistes en Bretagne. À plusieurs reprises, des communiqués de presse, disponibles sur le site web, leur sont envoyés. Ce point particulier montre toute l'énergie investie par Bzh-NY pour obtenir une conférence de presse. La conférence de presse constitue une épreuve pour le collectif car les articles de presse qui en résultent affirment la présence dans l'opinion publique en Bretagne. Ici, la façon dont sont utilisés les communiqués de presse pour entrer dans les réseaux de journalistes est similaire à la façon dont les dossiers de sponsoring sont utilisés pour identifier des partenaires économiques.

\subsection{Un collectif orienté vers le pays d'origine}

Le défilé en préparation est repris par Bzh-NY comme un événement devant paraître dans la presse et ainsi toucher le "grand public " (Boltanski et Thévenot, 1991) breton. Dans les quelques mois qui précèdent la venue du groupe breton à New York, le collectif Bzh-NY semble être partagé entre, d'un côté, l'organisation d'événements en mode projet (concerts, hébergement et restauration des artistes...) et, d'un autre côté, la recherche de notoriété en Bretagne, la communication autour du projet servant directement la notoriété du collectif dans sa région d'origine.

Nous ne prétendons pas ici être exhaustif, tant les éléments mis en place pour l'organisation d'un événement sont nombreux (réunions, discussions, communication dans les rues...). Notons néamoins que les acteurs " composent » (Callon, 1998) avec des éléments hétérogènes un ensemble qui répond à l'action en commun. Les technologies sélectionnées constituent des couplages forts avec certains usagers : Éric avec le forum, Obala avec Skype, SP avec Viadéo pour BZH Network.

En Bretagne, Bob essaie d'intéresser les réseaux économiques par le biais des dossiers de sponsoring qu'il remet en mains propres à quelques industriels. Cette démarche est reprise lorsqu'Obala fait parvenir des invitations officielles à des personnalités en Bretagne, ou lorsque les communiqués de presse sont envoyés aux journalistes. La mise en forme des connaissances est différente selon le réseau ciblé. À chaque réseau professionnel (journalistes, chefs 
d'entreprises, élus...), Bzh-NY fait correspondre un support doté de capacités de circulation et de visibilité particulières. Les communiqués de presse se retrouvent sur le site web, mais pas les dossiers de sponsoring ni les invitations officielles.

Alors que la phase d'exploration créait du lien autour d'une identité partagée, cette nouvelle phase cristallise la relation avec le pays d'origine et ses représentants politiques et économiques. Les technologies utilisées pour communiquer entre Bretons expatriés ne sont pas les mêmes que celles utilisées pour communiquer avec les représentants du pays d'origine. Avec cette nouvelle phase, un nouveau mode de structuration de l'expérience collective à l'étranger prend forme. L'inscription dans le débat public positionne Bzh-NY dans ce que Stéphane Dufoix considère comme un "mode centropériphérique » (Dufoix, 2003, p.72). Les institutions du pays d'origine jouent alors un rôle central pour intégrer les populations hors frontières.

\section{Animer une communauté, créer du lien fort}

\subsection{La cérémonie ou l'événement domestique}

Nous venons de voir deux phases d'activités que traverse un réseau de connaissances diasporiques (Meyer, 2007) et nous avons pu observer comment des systèmes techniques différents prennent place dans ces activités. Dans la phase d'exploration, les acteurs scrutent et explorent des liens à la recherche de nouveaux contacts et projets. Dans la phase de recherche de notoriété, l'organisation d'un événement vient servir le collectif pour affirmer son existence et sa relation avec la Bretagne.

En milieu d'année 2007, Bzh-NY officialise son existence par le dépôt d'un statut officiel d'organisation à but non lucratif (non profit organization). Un bureau est élu et un système de cotisation permet aux membres adhérents de participer à des tarifs préférentiels aux activités de l'association. Entre mai 2007 et août 2008, Bzh-NY organise ou participe à une douzaine de manifestations. L'alliance française de New York organise tous les ans un événement pour la commémoration de la prise de la Bastille, le 14 juillet. À cette occasion, en 2007, Bzh-NY vend des produits bretons dans les rues de Manhattan. En mai 2008, pour la Saint-Yves, fête de la Bretagne, l'association Bzh-NY organise un concert, ainsi qu'un "Fest $\mathrm{Noz}^{10}$ " à Times Square. À côté de ces événements visibles, l'association Bzh-NY organise des soirées et des repas dans les pubs et restaurants français de New York.

\footnotetext{
${ }^{10}$ Littéralement fête de nuit, le fest-noz est caractérisé par des danses et musiques bretonnes.
} 
Les TIC dans les collectifs diasporiques : étude des Bretons à New York

En ce qui concerne l'usage des TIC, cette troisième phase change radicalement. Les événements réguliers (tournois de belote, repas de Noël, pique-nique...) regroupent plusieurs générations de Bretons. Les nombreuses photographies disponibles sur le site web montrent parfois le soin apporté aux apparats du corps, habillement et bijoux. Le repas de Noël en constitue le meilleur exemple avec la présence des enfants. Les convives s'offrent des cadeaux. Deux articles présents sur le site web montrent à quel point les membres de l'association sont affables avec les visiteurs, sportifs ou artistes bretons accueillis avec respect. Les événements ainsi présentés s'apparentent à des cérémonies. Tous ces éléments permettent d'inscrire cette phase dans un mode de justification en public dont les valeurs correspondent à la «cité domestique »(Boltanski et Thévenot, 1991). En d'autres termes, la fête, les relations mondaines avec la famille élargie des Bretons de New York laissent entrevoir un collectif qui agit au nom de la tradition et de la famille.

\subsection{Les outils de l'animation}

\subsubsection{Le site web}

Suite à la semaine de festivités organisée pour Kevrenn Alré en mars 2007, peu de changements surviennent sur le site web. La communication de l'association est régulière au travers des newsletters. Pourtant, bien que la nature des activités ait changé, celles-ci continuent d'être traitées sur le mode événementiel. Les manifestations sont annoncées sur le site web et par la newsletter. Après chaque événement, des photos sont publiées dans une galerie publique sur le site web. Les fonctions dynamiques du site web qui permettaient l'inscription et le paiement en ligne sont progressivement abandonnées. Ces fonctions venaient aider les organisateurs à faire face à l'incertitude quant à la participation. Leur suppression indique une plus forte régularité des participants qui deviennent dès lors des habitués.

\subsubsection{Les forums}

Dans la première phase d'activité, le collectif Bzh-NY était très présent sur plusieurs forums francophones de New York. Alors que la seconde phase a montré une activité en retrait sur ces outils, cette troisième phase se caractérise par un retour sur ces espaces de libre échange. Mais les nombreuses discussions que l'on trouvait dans la période de construction du réseau ne refont pas leur apparition. Les messages déposés sur les forums suscitent peu d'échange. Le style des messages a lui aussi beaucoup changé. Ce n'est plus 
Éric qui intervient et les messages déposés s'apparentent à des annonces ou communiqués rédigés dans un style très formaté.

\subsection{Une communauté familiale}

La dernière phase d'activité du collectif de la diaspora bretonne à New York, observée entre mars 2007 et novembre 2008, rejoint les modèles communautaires. Un groupe stabilisé de personnes situées dans une localité proche entretient des relations durables et amicales autour d'activités conviviales. L'association inscrit dans ses statuts la distinction entre les acteurs (noyau dur) et son public (membres actifs). La régularité des événements et des participants est propice à la création de liens de confiance (Wenger, McDermott et Snyder, 2002). Le site web, construit comme un outil de relations publiques, s'aligne relativement bien avec ces nouveaux statuts. Alors que le site web s'adressait principalement aux partenaires de Bretagne, dorénavant, la cible principale du site web est le public qui participe aux différentes réunions. Les activités réalisées par Bzh-NY deviennent similaires à celles que réalisait l'Association bretonne des années 1960. Sur une note du site web qui accompagne les photos du repas de Noël de décembre 2007 il est précisé : "C'est avec plaisir que le président LC a pu constater la forte présence des anciens de Gourin ${ }^{11}$ ".

Précisons que Gourin est une commune rurale du Morbihan qui a connu des vagues d'expatriation vers les États-Unis et est devenue aujourd'hui un symbole des relations entre les Bretons et la ville de New York. Par ce message, le président élu de l'association Bzh-NY met en avant le fait que les différentes générations de Bretons vivant à New York se connaissent et cohabitent au sein d'une même communauté. Si l'on se réfère à nouveau aux "modes de structuration de l'expérience collective à l'étranger » définis par Stéphane Dufoix (Dufoix, 2003), Bzh-NY peut dorénavant être considéré comme étant dans un mode enclavé. C'est-à-dire que le collectif est une organisation locale qui se désintéresse du référent originel territorial.

\footnotetext{
${ }^{11}$ Disponible à l'adresse http://Bzh-ny.org/EN/images/0712 Nedeleg/nedeleg.php, consultée le 24 janvier 2009.
} 


\section{Conclusion}

De mars 2006 à novembre 2008, le collectif de la diaspora bretonne à New York traverse trois phases d'activités bien distinctes. Pour chacune s'opère une configuration propre des outils techniques et des activités. Nous proposons de représenter ces phases sous la forme du schéma suivant :

\section{Création d'un réseau}

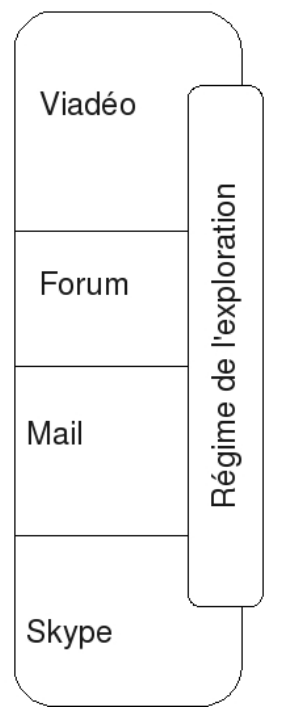

\section{Organisation d'un événement}

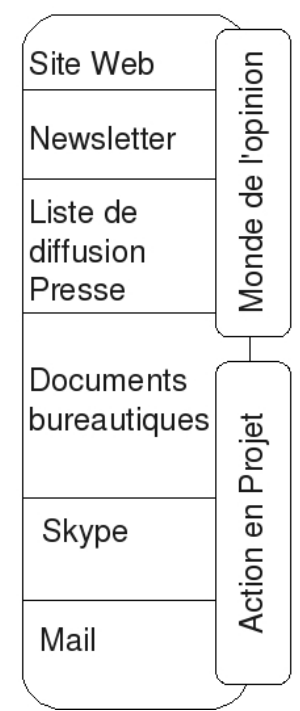

\section{Animation d'une communauté}

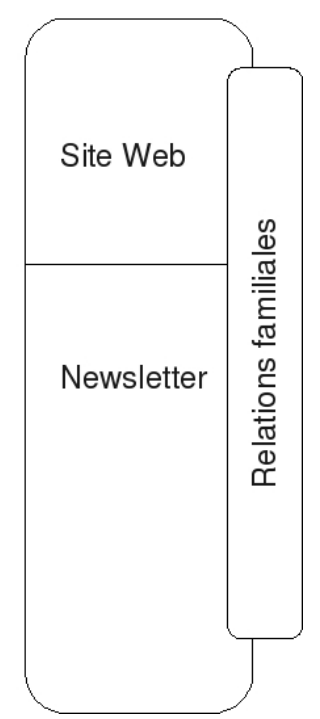

Figure 3 : L'alignement des outils techniques et de l'activité d'un réseau de connaissances diasporiques

Notre schéma est volontairement dépourvu d'axe temporel car nous ne pensons pas qu'il y ait un quelconque lien de succession généralisable entre les phases.

1 Dans la phase de création de réseau, ce sont quelques personnes qui tissent un ensemble très large de relations. Une grande partie de l'énergie fournie pour tisser ce réseau provient sans conteste d'Obala pour qui l'attachement à la Bretagne est mêlé à un projet professionnel. Dans cette phase, le web prend une part très importante, même si 
quelques rencontres ont lieu dans la vie réelle. Dans cette configuration, les quatre outils internet orientent tout autant qu'ils supportent l'exploration.

2 La phase d'organisation d'un événement voit un regroupement d'acteurs se coordonner en vue de mener à bien un projet. L'agrégation d'outils hétérogènes porte la visibilité de l'événement. Cette fois-ci, l'extension de réseau est orientée vers la recherche de partenaires institutionnels et économiques.

3 Enfin, dans la phase d'animation de communauté, on observe une forme de repli géographique et social du collectif. II consolide les liens existants et cherche moins à s'étendre. Sur le web, le site devient une mémoire du collectif qui s'adresse à la fois au public des participants (la grande famille des Bretons de New York) et à un public beaucoup plus large (les visiteurs du web).

En prenant les régimes d'engagement comme point d'entrée, on identifie de nettes configurations d'outils pour chaque type d'activités. Le régime de l'exploration s'adapte particulièrement bien aux outils de l'internet dont les fonctions de base sont la recherche et la rencontre. Le régime de l'action en plan recoupe une composition fine d'outils de coordination en équipe. Tous ces outils ne donnent pas lieu à une visibilité élargie et ne positionnent pas un public observateur comme le font le site web et la newsletter. Dans les deux dernières phases, ces outils techniques renvoient à un mode de justification en public.

Cette observation précise la place du régime d'exploration sur l'internet. Nous pensons que ce régime, même s'il s'y prête particulièrement bien, ne domine pas l'ensemble des usages de l'internet. Dans notre exemple, le site web de Bzh-NY positionne le collectif en situation de justification face à « celui qui va arriver » (Thévenot, 2006, p.107), c'est à dire un public potentiel. On voit donc comment une technologie particulière, ici le site web, crée une tension entre les activités du collectif et leur mise en visibilité sur le web. II serait donc intéressant d'identifier des réseaux de connaissances diasporiques qui utilisent des outils du web 2.0 pour voir comment la présence d'un public est prise en compte. 


\section{Références bibliographiques}

AURAY N., 2005, Une autre façon de penser le lien entre technique et politique. Le réagencement de l'activité autour de l'exploration, Document de travail, dans le cadre du programme GSPM-EHESS "Politiques du proche", coordonné par Laurent Thévenot.

BOLTANSKI L. et L. THÉVENOT, 1991, De la justification, les économies de la grandeur, Paris, Gallimard.

BOLTANSKI L. et E. CHIAPELLO, 1999, Le nouvel esprit du capitalisme, Paris, Gallimard.

BOULLIER D., 2008, « Politiques plurielles des architectures d'internet », Cahier Sens Public, L'internet entre savoirs, espaces publics et monopoles, $n^{\circ} 6 / 7$, octobre.

BOULLIER D., S. LE BAYON et F. PHILIP, à paraître, « Formats techniques, formats communautaires et formats d'engagement. Le cas d'une communauté diasporique ", dans F. MILLERAND, S. PROULX et J. RUEFF (dir), Le Web relationnel, mutation de la communication ?, Québec, Presses de l'Université du Québec.

BORZEIX A. et F. COCHOY, 2008, «Travail et théories de l'activité : vers des workspace studies ? », Sociologie du travail, n50, pp.273-286.

CALLON M., 1986, "Éléments pour une sociologie de la traduction. La domestication des coquilles Saint-Jacques et des marins-pêcheurs dans la baie de Saint-Brieuc », L'année sociologique, n³6, pp.169-208.

CALLON M., 1998, The laws of the markets, Oxford, Blackwell.

DIMINESCU D., 2005, «Le migrant connecté. Pour un manifeste épistémologique », Migrations/Sociétés, vol.17, n¹02, pp.275-292.

DUFOIX S., 2003, Les diasporas, Paris, Presses universitaires de France.

GRANOVETTER M. 1973, "The strength of weak ties », American Journal of Sociology, vol.78, n6, pp.1360-1380.

JOUAS J., C. LE CORRE et C. JAMET, 2005, Ces Bretons d'Amérique du Nord, Ouest-France.

LATOUR B., 2006, Changer de société. Refaire de la sociologie, Paris, La Découverte. 
MEYER J.-B., 2007, Building sustainability : The new frontier of diaspora knowledge networks, COMCAD Working Papers, vol.35.

THÉVENOT L., 2006, L'action au pluriel, Sociologie des régimes d'engagement, Paris, La Découverte.

WENGER E., R. MCDERMOTT et W. SNYDER, 2002, Cultivating communities of practice: A guide to managing knowledge, Boston, Harvard Business School Press. 